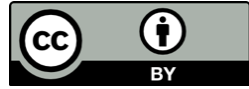

\title{
La situación de las PYMEs en un contexto de post pandemia
}

Proposal for Emotional Competencies and conflict resolution in university students

Proposta de Competências Emocionais e Resolução de Conflitos em Estudantes Universitários.

\section{ARTÍCULO GENERAL}

\author{
Evelyn Elizabeth Rojas Mayta \\ evelyn_contabilidad@hotmail.com \\ https://orcid.org/0000-0001-8297-915X \\ CECAGEM EIRL, Lima - Perú
}

\author{
Marcos Martin Bustios Martinez \\ mbustios@continental.edu.pe \\ https://orcid.org/0000-0002-0165-7026 \\ CECAGEM EIRL, Lima - Perú
}

Recibido 16 de Diciembre 2020 | Arbitrado y aceptado 06 de Enero 2021 | Publicado el 01 de Marzo 2021

\section{RESUMEN}

Durante el año 2020 la humanidad entera tuvo que enfrentarse a una pandemia, por primera vez ocasionada por un elemento no económico ni social sino de salud. Sin embargo, la propagación del denominado Covid-19, afectó incluso a las economías más poderosas, lo que implicó que en el resto de los mercados también se sufrieran consecuencias profundas. En ese sentido, las PYMEs, fueron una de las empresas más afectadas por la falta de formalidad, procesos estandarizados, materiales, insumos, entre otros aspectos, que devinieron en el cierre de muchas de ellas. La perspectiva a futuro es un panorama que, aunque incierto, ya deja ver determinados cambios en elementos como la demanda, el tipo de pago, modalidad de trabajo, y marcos legales. En ese sentido, el presente artículo se planteó reflexionar sobre los efectos que ha tenido en las PYMEs la pandemia de Covid-19 así como exponer aquellos elementos que más cambios han sufrido generando nuevas dinámicas económicas en este sector.

Palabras clave: Pymes, post pandemia, Covid-19.

\section{ABSTRACT}

During the year 2020, humanity as a whole had to face a pandemic, for the first time caused by an element that was not economic or social, but health. However, the spread of the so-called Covid-19 affected even the most powerful economies, which meant that the rest of the markets also suffered profound consequences. In this sense, SMEs were one of the companies most affected by the lack of formality, standardized processes, materials, inputs, among other aspects, which led to the closure of many of them. The future perspective is a panorama that, although uncertain, already reveals certain changes in elements such as demand, the type of payment, work modality, and legal frameworks. In this sense, this article was proposed to reflect on the effects that the Covid-19 pandemic has had on SMEs, as well as to expose those elements that have suffered the most changes, generating new economic dynamics in this sector.

Keywords: SMEs, post pandemic, Covid-19.

\section{RESUMO}

Durante o ano de 2020, a humanidade como um todo teve que enfrentar uma pandemia, pela primeira vez causada por um elemento que não era econômico ou social, mas a saúde. No entanto, a propagação do chamado Covid-19 afetou até as economias mais poderosas, o que fez com que os restantes mercados também sofressem consequências profundas. Nesse sentido, as PMEs foram uma das empresas mais afetadas pela falta de formalidade, padronização de processos, materiais, insumos, entre outros aspectos, o que levou ao fechamento de muitas delas. A perspectiva futura é um panorama que, embora incerto, já revela algumas mudanças em elementos como a demanda, o tipo de pagamento, a modalidade de trabalho e os marcos legais. Neste sentido, este artigo foi proposto para refletir sobre os efeitos que a pandemia de Covid-19 teve nas PME, bem como expor aqueles elementos que mais sofreram alterações, gerando novas dinâmicas económicas neste setor.

Palavras-chave: PME, pós-pandemia, Covid-19. 


\section{Introducción}

La humanidad a través de milenios se ha tenido que enfrentar a innumerables obstáculos masivos como desastres naturales, desastres producidos por los propios seres humanos, plagas y enfermedades (Martínez-Taboas, 2020) sin embargo, los países en el mundo no estaban preparados (ni siquiera los más poderosos) para la más reciente pandemia mundial ocasionada por la Covid-19 (Ferrer, 2020).

La propagación del nuevo coronavirus, causante de la enfermedad conocida como Covid-19, dio pase a una gigantesca crisis sanitaria que a su vez dio origen a una económica en todos los países del globo (Guimón, 2020, citado en Foladori y Delgado 2020); una crisis que ha tenido repercusiones sin precedentes en todos los aspectos de la vida de las personas como la comunicación, la producción, el comercio, el consumo, y en definitiva, también en el trabajo (Tembo, 2020). Para Rosales (2020) esta sería la primera crisis económica que no ha sido originada por causas políticas, económicas o culturales $\mathrm{y}$, sin embargo, su impacto es tan grande que ha afectado a todas las economías y mercados del mundo y con un nivel superior a cualquier otra crisis anterior.

La Covid-19 ha supuesto el cierre de 2,7 millones de empresas, equivalente al 19\% de empresas en la región latinoamericana y la pérdida de trabajo de más de 8,5 millones de personas (CEPAL, 2020 citado en Aguirre et al., 2020). Dentro de esas cifras, el principal porcentaje de empresas perjudicadas son las pequeñas y medianas empresas, es decir, las PYMEs.

Por todo lo expuesto, se pretende con la presente investigación reflexionar sobre los efectos que ha tenido en las PYMEs el complejo momento que ha vivido la humanidad, caracterizado principalmente por la pandemia de Covid-19 así como exponer aquellos elementos que más cambios han sufrido generando nuevas dinámicas económicas en este sector.

\section{Metodología}

La búsqueda de la información para la revisión de literatura se hizo consultando las siguientes bases de datos como son Proquest, Scopus y Google Scholar para encontrar revistas del más alto nivel científico. Asimismo, cabe mencionar que para la realización 
de lo mencionado se emplearon también palabras clave como como PYMEs, SME's, Pandemia, Covid-19, Confinamiento.

Cada uno de estos descriptores se combinaron entre sí durante la búsqueda utilizando los operadores boléanos “and” y “or". Además, algunos de los criterios o filtros que se utilizaron para hacer muchos más específica la búsqueda y ser más precisos con los documentos encontrados en las diferentes bases de datos consultadas fueron: que sean artículos de revistas, y la antigüedad de la publicación que no sea mayor a 5 años.

Toda la información previa relacionada a los filtros aplicados y las especificaciones sirvieron como criterios de inclusión, es decir que sean artículos publicados en revistas científicas, que no excedan los cinco años de antigüedad, que la temática guarde relación con las variables de PYMEs y post pandemia. Se excluyeron, en tanto, todos los documentos que no respetaran lo postulado así como aquellos que estaban incompletos o que tenían enlaces averiados.

\section{Resultados y discusión}

\section{a. Pandemia y Covid 19}

En diciembre de 2019 se reportaron una serie de casos de pacientes hospitalizados con una enfermedad nueva caracterizada por neumonía e insuficiencia respiratoria, a causa de un nuevo coronavirus (SARS-CoV-2), en la provincia de Hubei, China. Posterior a ello, en febrero de 2020 la Organización Mundial de la Salud (OMS) ya había nombrado a este agente etiológico como COVID-19 (Coronavirus Disease, 2019) (Ferrer, 2020).

Pese a las medidas de contención, la fuente previa indica que la enfermedad traspasó fronteras y siguió avanzando hasta afectar al resto de los países de Asia, Medio Oriente y Europa. Fue así como el 11 de marzo, la OMS declaró a esta enfermedad como una pandemia mundial. El anuncio se dio en una rueda de prensa transmitida a todo el globo que lideró Tedros Ghebreyesus, director general de la entidad.

Pese a que aún no se ha confirmado el origen de esta enfermedad y los debates respecto a esta son innumerables, un hecho certero es que se trata de una crisis manifiesta de salud que alcanza, aunque en diferente grado, a todas las clases sociales, grupos étnicos, ideologías y sexos (Foladori y Delgado, 2020). Esta enfermedad trajo consigo no solo millones de muertes alrededor del mundo sino consecuencias indirectas pero 
relacionadas con otros temas. Ello se debe a que las pandemias están caracterizadas por la confusión, temores, incertidumbre y la probabilidad de muertes en seres queridos. Asimismo, se relacionan con innumerables estresores sociales tales como hacer ajustes en las rutinas, separación de amigos y familiares, pérdida de empleos y aislamiento social (Martínez-Taboas, 2020).

Todos los analistas económicos y financieros concuerdan en que la crisis de salud generada por la propagación del Covid-19 dio pase, a su vez, a una crisis económica en todos los países del mundo (Guimón, 2020, citado en Foladori y Delgado 2020) que se suma a los problemas sociales preexistentes como la desigualdad, los altos índices de pobreza, desempleo y precariedad laboral.

\section{b. Crisis económica mundial}

La Organización Internacional de Trabajo - OIT (2020) estimó que la crisis económica, derivada de la crisis sanitaria por la Covid-19, destruiría alrededor de 305 millones de empleos en el mundo. Asimismo, la pandemia generó un contraste amplio entre lo indicado por el Fondo Monetario Internacional (FMI), entidad que, a enero de 2020 esperaba un crecimiento positivo de la renta per cápita en más de 160 países, sin embargo, para abril se estimó que más de 170 países experimentarán un crecimiento negativo (Georgieva, 2020).

Las Américas son en la actualidad las más afectadas por la pérdida de tiempos de trabajo, estimadas en 18,6 \%, seguido por Europa con un $14.3 \%$, Asia con un 13,4 \%, Medio Oriente con el 13,5 \% y el continente Africano con un 12,7 \% (Ponce et al. 2020).

Cabe mencionar otros datos relevantes como que la tasa de pobreza en la región latinoamericana crecería en un 4,4\% durante 2020 pasando de un 30,3\% a 34,7\%, cifras que representan a 29 millones de personas que quedarían en situación de pobreza. En tanto, la pobreza extrema aumentaría en un 2,5\% yendo del $11,0 \%$ al $13,5 \%$, reflejando el estado al que pasarían 16 millones de personas. Finalmente, la tasa de desempleo se ubicaría en $11,5 \%$, un aumento de 3,4 puntos porcentuales respecto al nivel de 2019 , que era del $8,1 \%$. De esta forma, el número de desempleados de la región llegaría a 37,7 millones (Richard y Fushimi, 2020). 
De forma más específica, esta crisis generó el cierre de 2,7 millones de empresas, equivalente al 19\% de empresas en la región latinoamericana y la pérdida de trabajo de más de 8,5 millones de personas (CEPAL, 2020 citado en Aguirre et al., 2020). El principal porcentaje de empresas perjudicadas son las pequeñas y medianas empresas, es decir, las PYMEs.

\section{c. Impacto económico en las PYMEs}

La Organización Internacional de Trabajo señaló una serie de quiebras en las PYMEs como consecuencia de la crisis económica, así también se estimó que más del $50 \%$ de estas empresas no lograrían sobrevivir al 2020 (OCDE, 2020).

La Encuesta sobre el Impacto Empresarial de la COVID-19 del Centro de Comercio Internacional - ITC, ha recopilado datos sobre cómo ha afectado la pandemia a 4467 empresas de 132 países, de tal forma que se puede ejemplificar mejor estas consecuencias (Figura 1 y 2)

Figura 1. Empresas afectadas por la pandemia

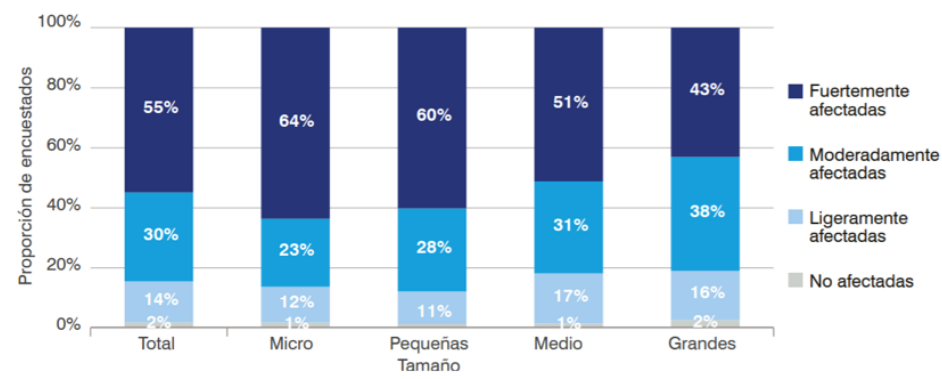

Fuente: Tembo (2020).

Figura 2. Cierre de Pymes por pandemia

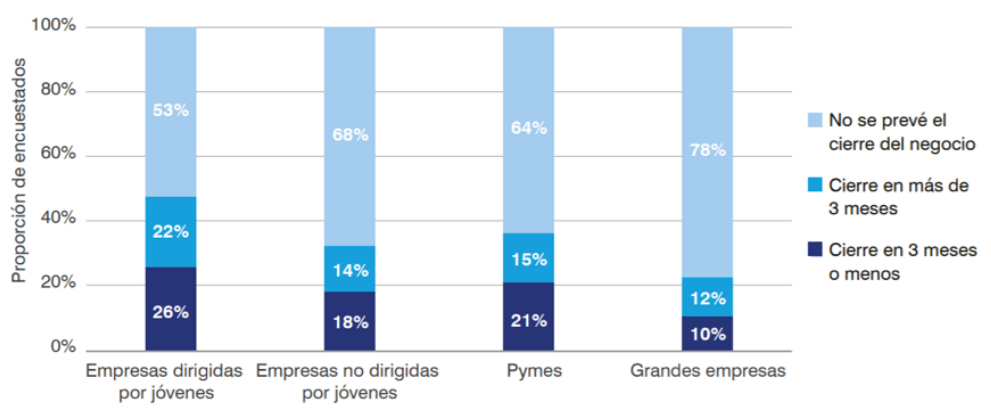


Fuente: Tembo (2020).

Lamentablemente desde que se decretaron las medidas de confinamiento prácticamente en todos los países del mundo, tales como distanciamiento social, uso de mascarillas y protectores faciales, así como la limitación de desplazamientos y restricción de vehículos particulares, las PYMEs se vieron afectadas.

La pandemia y Covid-19 suponen un reto gigante para estas empresas, que en la mayoría de los casos han tenido o tendrán que adaptar sus establecimientos y el trabajo de sus empleados y empleadas a las medidas de seguridad establecidas para evitar contagios. En algunos casos incluso se verán obligadas a reducir su actividad o a solicitar ayudas financieras para hacer frente a esta situación (Naciones Unidas, 2020).

Otros factores más personales como la desconfianza de los consumidores ha generado graves pérdidas en las ventas de las empresas, volviendo insostenible sus aparatos productivos y financieros, muchas incluso llegaron al colapso. En ese sentido, trabajadores y empresas están frente a una catástrofe, y es preciso que se actúe con rapidez, decisión y coordinación (Ponce et al. 2020) para que estas se adecúen al contexto actual y planifiquen cambios para sobrevivir en los meses que siguen luego de la pandemia.

\section{d. PYMEs}

El desarrollo de la PYME (Pequeña y Micro Empresa) y del sector informal urbano en el Perú ha sido un fenómeno característico de las últimas dos décadas, debido al acelerado proceso de migración y urbanización que sufrieron muchas ciudades (Arbulú y Otoya, s.f).

Figura 3. PYMEs según personal y ventas en el MTPE

\begin{tabular}{|c|c|c|}
\multicolumn{3}{|c}{ Ministerio de Trabajo } \\
\hline Tamaño de Empresa & Personal Ocupado & Ventas Anuales \\
\hline Microempresa & Hasta 10 & Hasta 12 UIT \\
\hline Pequeña empresa & De 11 a 20 & De 13 a 25 UIT \\
\hline
\end{tabular}

Fuente: Herrera, 2011.

Las PYMEs, en el Perú, son organizaciones económicas básicas, producto de los emprendedores; y que debido al poco o mulo apoyo del Estado, operaran mayormente en 
la informalidad (López, 2020). Cabe mencionar que en países en vías de desarrollo como el Perú, las PYMEs cumplen un rol fundamental en la dinámica del mercado, produciendo y ofertando bienes, añadiendo valor agregado y contribuyendo a la generación de empleo (Cacho et al. 2019).

Así, el sector conformado por las PYMEs posee una gran importancia dentro de la estructura industrial de nuestro país, tanto en términos de su aporte a la producción nacional (42\% aproximadamente según PROMPYME) como de su potencial de absorción de empleo (cerca de 88\% del empleo privado según PROMPYME2 (Arbulú y Otoya, s.f)

\subsubsection{PYMEs Post Pandemia}

Siendo las pequeñas y medianas empresas, PYMEs, un tipo de organización que, si bien nutren buena parte del tejido empresarial de todos los países, independientemente del tamaño de sus economías, ellas adolecen de dirección y estructuras internas con capacidades suficientes para enfrentar los efectos económicos de una pandemia con las dimensiones del COVID 19, convirtiéndolas en entidades altamente vulnerables (Rosales, 2020).

En los países en desarrollo, estas empresas se han visto gravemente afectadas, pese a que las pequeñas empresas son competitivas y resilientes, muchas de ellas se han visto sacudidas por profundas perturbaciones en las cadenas de suministro internacionales (Tembo, 2020).

Asimismo, si bien la Covid-19 no va a tener repercusión en algunas tendencias firmemente arraigadas en las dinámicas propias del mercado, es evidente que ya hay nuevas tendencias que están configurando un nuevo entorno en el cual se desenvolverán las organizaciones empresariales (Rosales, 2020).

\subsubsection{Elementos que están cambiando}

Aguirre et al (2020) y Rosales et al (2020) enlistan algunos elementos que están viéndose alterados con el paso de la pandemia por la Covid-19 a nivel mundial y de manera enfática en la dinámica de las PYMEs. Estos son: 
- Demanda

Un primer caso de la metamorfosis es el elemento de la demanda. Cabe indicar que en los últimos años no sólo ha cambiado la forma en que compramos, utilizando de forma creciente los canales digitales, sino también los hábitos de consumo han variado, tanto en cantidad y frecuencia como en marcas elegidas. La Covid-19 ha profundizado en estos aspectos haciendo más común que el ciudadano opte por nuevas marcas, en nuevos sitios web (algunos incluso que no existían antes de la cuarentena), y consumiendo en los locales más cercanos a su vivienda.

- Pago

La forma de pago también está cambiando fuertemente a causa de la pandemia, así, el uso de las tarjetas de débito y crédito, como también de forma incremental al método de pago digital a través de aplicaciones en el celular, evitando de esta forma todo tipo de contacto entre comprador y vendedor es una práctica cada vez más común. Incluso en algunos establecimientos es ya una regla este tipo de intercambios de dinero sin contacto físico.

- Normas y legalidad

Las nuevas formas de trabajo, así como las nuevas políticas de compra, entre otras nuevas actividades, requieren que el sistema judicial rápidamente actúe para no provocar más vacíos legales precisamente en estas problemáticas. Así, se requieren cambios en el formato jurídico institucional y operacional del Estado, en particular en lo referente a la prestación de servicios básicos de educación y salud, incluyendo lo relativo al financiamiento de estos servicios.

- Fuerza de trabajo

No sólo se debe empoderar a los empleados, producto de la distancia entre pares y consecuentemente la ruptura de canales de comunicación imperantes hasta fines de 2019 , sino también el control sobre la fuerza laboral ha debido cambiar de uno netamente presencial (visual), a un control sobre el resultado de la tarea de forma digital. El teletrabajo se volvió una norma a raíz de la pandemia y sobre este tema se ahondará en el siguiente ítem. 


\subsubsection{Rol de las TIC y el teletrabajo}

La digitalización es clave en un escenario como el actual en el que millones de personas están obligadas a trabajar desde sus casas, los eventos y reuniones presenciales han sido sustituidos por los virtuales, y el comercio electrónico está tomando un impulso sin precedentes (Naciones Unidas, 2020). Asimismo, la digitalización no solo es una herramienta que le va a permitir a las PYMEs adaptarse a una crisis como la que se está viviendo, sino que puede suponer un impulso para fomentar la sostenibilidad dentro de las organizaciones y una cultura de desarrollo sostenible a largo plazo.

Ponce et al. (2020) señala que los modelos de negocios han tenido que cambiar drásticamente, dando lugar a elementos clave como la flexibilidad laboral, el uso de medios tecnológicos y el teletrabajo que han dado una alternativa para seguir operando y que las empresas puedan mantenerse en contacto con sus clientes.

En ese sentido, gracias a los procesos de digitalización y el rol de las Tecnologías de Información y Telecomunicación (TIC) durante la pandemia por la Covid-19, se fomentó, y en algunos casos se instauró de forma obligatoria, la modalidad del teletrabajo. Este se refiere a la realización habitual de la actividad laboral a distancia en un lugar distinto al habitual en la empresa, y a veces en horario diferente (Peiró y Soler, 2020).

Figura 4. Población ocupada que puede teletrabajar por sector de actividad

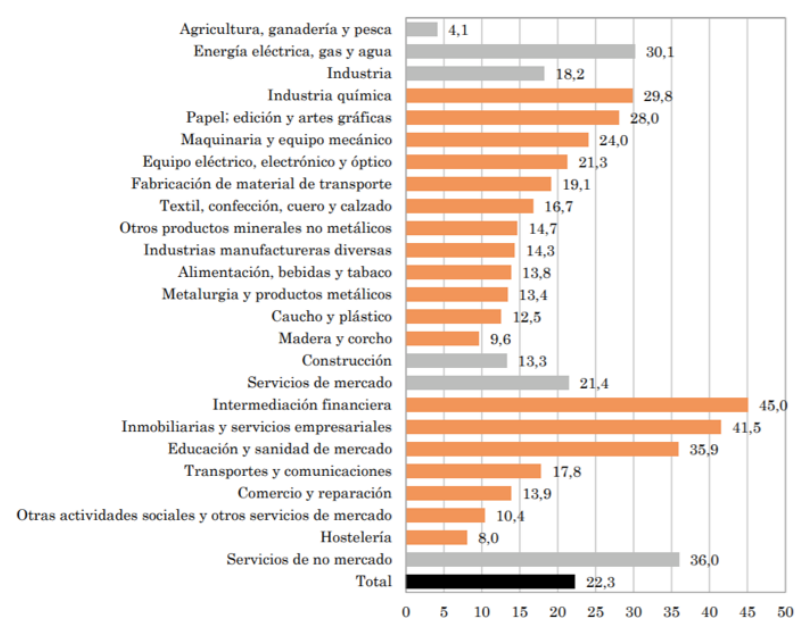

Fuente: INE, Randstad (s.f) citado por Peiró y Soler (2020) 
Existe un importante potencial para la práctica del teletrabajo que hasta hace pocas semanas se utilizaba mucho menos que durante la Covid-19 (Peiró y Soler, 2020). La expansión y generalización del teletrabajo, que hasta la actualidad había experimentado un discreto desarrollo, en estas circunstancias, ha sido uno de los pilares de contención de la recesión económica pudiendo favorecer a la continuación de las empresas (Fernández, 2020).

En ese sentido, las PYMEs deben demostrar que tienen una capacidad extraordinaria para adaptarse a las nuevas tendencias de mercado y alinear su producción y comercialización de bienes y servicios a las nuevas necesidades en panoramas complejos.

\section{Referencias}

Arbulú, J. y Otoya, J. (s.f) La PYME en el Perú: características e importancia de la pyme en nuestra economía. CENDOC, ESAN. http://cendoc.esan.edu.pe/fulltext/ejournals/PAD/7/arbulu.pdf

Aguirre, R., Rusconi, J., Turano, C., Todesca, A. y Zapata, L. (2020) Covid-19 y su impacto en las Pymes argentinas: Perspectivas para América Latina. Palermo $\begin{array}{llll}\text { Business } & \text { Review, } & \mathrm{N}^{\circ} & 22,\end{array}$ https://www.palermo.edu/negocios/cbrs/pdf/pbr22/PBR_22_12.pdf

Cacho, A., Carrasco, Y. y Bacalla, M. (2019) El desconocimiento de las normas y su afectación en las PYMES. Revista Pakamuros, 7, 2, 89-98.

CEPAL. (2020). Euro Mi Pyme - COVID-19. https://www.cepal.org/es/euromipyme/ mipymes-covid-19

Ferrer, R. (2020) Pandemia por COVID-19: el mayor reto de la historia del intensivismo. Medicina Intensiva, 44(6), 323-324.

Fernández, L. (2020). El teletrabajo de actor secundario a protagonista en el escenario del COVID-19. La Ley, ISSN 1989-6913, № 9708.

Foladori, G. y Delgado, R. (2020) Para comprender el impacto disruptivo de la covid-19, un análisis desde la crítica de la economía política. MIGRACIÓN y DESARROLLO, volumen 18 , número $34,161-177$. 
Georgieva, K. (2020). Confronting the Crisis: Priorities for the Global Economy. Fondo Monetario Internacional. https://www.imf.org/en/News/Articles/2020/04/07/ sp040920-SMs2020-Curtain-Raiser

Guimón, P. (2020) El FMI pronostica para este año la mayor recesión desde la Gran Depresión de 1929. El País. https://elpais.com/economia/(2020)-04-09/el-fmipreve-que-la-pandemia-provocara-el-mayor-impacto-en-la-economia-desdelagran-depresion.html

Herrera, B. (2011) Análisis estructural de las MYPEs y PYMEs. Quipukamayoc. Revista de la Facultad de Ciencias Contables, Vol. 18 N. ${ }^{\circ} 35$ pp. 69-89.

López, V. (2020) Emprendedores y pymes en el Perú. Economía \& Negocios, Vol. 02 $\mathrm{N}^{\circ} 01$, pp. 11-14. http://www.revistas.unjbg.edu.pe/index.php/eyn/article/view/903/986

Martínez-Taboas, A. (2020). Pandemias, COVID-19 y Salud Mental: ¿Qué Sabemos Actualmente? Revista Caribeña de Psicología, 4(2), 143-152. https://doi.org/10.37226/rcp.v4i2.4907

Naciones Unidas (2020) Pymes y COVID 19: hacia una recuperación sostenible. Red Española del Pacto Mundial.

OCDE (2020). Coronavirus: SME policy responses. Secretary-General of the OECD. https://oecd.dam-broadcast.com/pm_7379_119_119680-di6h3qgi4x.pdf

Organización Internacional de Trabajo - OIT (2020) Observatorio de la OIT: El COVID19 y el mundo del trabajo. Cuarta edición. https://www.ilo.org/wcmsp5/ groups/public/@dgreports/@dcomm/documents/briefingnote/wcms_743154.pdf

Peiró, J. y Soler, A. (2020) El impulso al teletrabajo durante el covid-19 y los retos que plantea. Ivie y Universitat de València. https://umivale.es/dam/webcorporativa/Documentos-prevenci-n-y-salud/11.Covid19IvieExpress.El-impulsoal-teletrabajo-durante-el-COVID-19-y-los-retos-que-planteaf.pdf

Ponce, J., Palacios, D., Palma, A. y Salazar, G. (2020) Crisis económica pre y postpandemia: su incidencia en la mortalidad de las MiPymes en Ecuador. Revista 
Observatorio de la Economía Latinoamericana, ISSN: 1696-8352. https://www.eumed.net/rev/oel/2020/09/crisis-ecuador.html

Richard, E. y Fushimi, J. (2020) Pensando en la salida del aislamiento social, preventivo y obligatorio. Ideas para mitigar la crisis post-pandemia de covid-19. Estudios de Derecho Empresario. ISSN 2346-9404.

Rosales, R. (2020) La Pyme post COVID 19: ¿seguirá siendo un comodín entre el Estado y el Mercado? Revista Catalejo Virtual.

Tembo, D. (2020). COVID-19: el gran confinamiento y su impacto en las pequeñas empresas. Perspectivas de competitividad de las PYMEs. Centro de Comercio Internacional - $\quad$ ITC. https://www.intracen.org/uploadedFiles/intracenorg/Content/Publications/SMEC O2020/ITC_SMECO-2020ExSummary-Spanish.pdf 